\title{
Relação entre habilidades cognitivas não-verbais e variáveis presentes no contexto educacional
}

\author{
Tatiana Pontrelli Mecca \\ Centro Universitário FIEO - Osasco - SP \\ Tatiana Abraão Jana \\ Universidade Presbiteriana Mackenzie - São Paulo - SP \\ Mário Rodrigues Simões \\ Universidade de Coimbra - Coimbra - Portugal \\ Elizeu Coutinho de Macedo \\ Universidade Presbiteriana Mackenzie - São Paulo - SP
}

\begin{abstract}
Resumo
O objetivo deste estudo foi verificar a relação entre habilidades cognitivas não-verbais avaliadas pela Escala Internacional de Inteligência Leiter-R e variáveis presentes no contexto educacional. A Leiter-R foi administrada em 213 crianças com idades entre 6 e 8 anos, pertencentes a escolas públicas e particulares, juntamente com o Teste de Competência de Leitura de Palavras e Pseudopalavras e a Prova de Aritmética. Os alunos de escolas particulares alcançaram uma pontuação superior aos de escolas públicas nos 6 subtestes da Leiter-R, sendo que essas diferenças também foram observadas na faixa etária de 6 anos. No entanto, aos 7 e 8 anos, as diferenças foram apenas em 2 subtestes. As correlações entre a Leiter-R e os testes de desempenho acadêmico foram positivas, significativas e de magnitude moderada. As análises de regressão linear indicaram predição das habilidades cognitivas em $24,2 \%$ do desempenho em aritmética e $30,6 \%$ do desempenho de leitura.
\end{abstract}

Palavras-chave: Inteligência; educação; testes de desempenho.

\section{Relationship between non-verbal cognitive abilities and variables in the educational context}

\begin{abstract}
The aim of this study was to investigate the relationship between non-verbal cognitive skills evaluated by the Leiter-R Intelligence International Scale and variables in the educational context. The Leiter-R was administered to 213 children aged between 6 and 8 years old, belonging to public and private schools, along with the Reading Competence Test of words and pseudo words and Proof of arithmetic. Students of private schools reached a higher score to the public schools in six subtests of the Leiter-R, and these differences were also observed in the age group for six years. However, between the ages of 7 and 8 years, the differences were only in two subtests. The correlations between the Leiter-R and academic achievement tests were positive, significant and of moderate magnitude. The linear regression analyzes indicated prediction of cognitive abilities in $24.2 \%$ of the arithmetic performance and $30.6 \%$ of the read performance.
\end{abstract}

Keywords: Intelligence; education; performance tests.

\section{Relación entre habilidades cognitivas no-verbales y variables presentes en el contexto educacional}

\begin{abstract}
Resumen
El objetivo de este estudio fue verificar la relación entre habilidades cognitivas no-verbales evaluadas por la Escala Internacional de Inteligencia Leiter-R y variables presentes en el contexto educacional. Se administró la Leiter-R a 213 niños con edades entre 6 y 8 años, pertenecientes a escuelas públicas y particulares, juntamente con el Test de Competencia de Lectura de Palabras y Pseudo-palabras y la Prueba de Aritmética. Los alumnos de escuelas particulares alcanzaron una puntuación superior a los de escuelas públicas en los 6 sub-testes da Leiter-R, siendo que esas diferencias también fueran observadas en la etapa etaria de 6 años. Sin embargo, a los 7 y 8 años, las diferencias fueron sólo en 2 sub-testes. Las correlaciones entre la Leiter-R y los testes de desempeño académico fueron positivas, significativas y de magnitud moderada. Los análisis de regresión linear indicaron predicción de las habilidades cognitivas e nel $24,2 \%$ del desempeño en aritmética y el $30,6 \%$ del desempeño de lectura. Palabras clave: inteligencia; educación; testes de desempeño.
\end{abstract}




\section{Introdução}

A inteligência é um construto constantemente associado ao desempenho acadêmico. Vários estudos apontam correlações entre funcionamento intelectual e habilidades necessárias no contexto escolar (Gomes, 2010; Pereira, \& Almeida, 2010). Essas relações são relevantes para que a interpretação dos resultados dos testes de inteligência possa ter implicações acadêmicas. Neste sentido, justifica-se a realização de estudos que investiguem relações entre habilidades cognitivas e as variáveis presentes no contexto escolar.

Alguns instrumentos já normatizados para a população brasileira apresentam estudos ou dados normativosindependentes para indivíduos pertencentes a escolas públicas e particulares (Angelini, Alves, Custódio, Duarte, \& Duarte, 1999; Almeida, \& Primi, 2000; Alves,\& Duarte, 2001). Tal abordagem tem sido usada uma vez que estes grupos apresentam diferenças em seu desempenho. Desta forma, justifica-se a realização de estudos que comparem habilidades cognitivas entre crianças de escolas públicas e particulares.

Uma das diferenças importantes entre crianças de escolas públicas e particulares verificadas pelo IBGE é o nível de escolaridade das mães, que constitui um indicador importante do nível socioeconômico. Dados recentes indicam que há maior prevalência de mães de crianças pertencentes a escolas públicas que não têm instrução ou que não finalizaram o Ensino Fundamental. Por outro lado, há maior prevalência de mães de crianças pertencentes a escolas particulares que completaram o Ensino Médio e o Ensino Superior (Instituto Brasileiro de Geografia e Estatística [IBGE], 2012). Diversos estudos apontam que o funcionamento intelectual está associado ao nível educacional materno (Chevalier, 2005; Carneiro, Merghi, \& Parey, 2007; 2013).

Estudos apontam que a diferença de um ano a mais de escolaridade materna está associada a uma diferença de 5 pontos percentuais nos resultados dos seus filhos em testes de leitura e de matemática como o Peabody Individual Achievement Test. (Carneiro, Merghi, \& Parey, 2007; 2013). Vista e Grantham (2010) investigaram a influência do nível educacional dos pais na inteligência fluida dos filhos por meio do Naglieri Nonverbal Ability Test, um instrumento que avalia o raciocínio analógico. Esses autores observaram um aumento de cerca de 3 pontos no QI dos filhos de acordo com a mudança dos níveis de escolaridade dos pais: Ensino Médio, Graduação e Pós-graduação.

Além das variáveis socioeconômicas, a inteligência está associada ao sucesso acadêmico, como o desempenho em aritmética e leitura (Stock, Desoete, \& Roeyers, 2009; Lu, Weber, Spinath, \& Shi, 2011). Primi, Santos e Vendramini (2002) ressaltam que as habilidades aritméticase de leitura são fundamentais para o processo de escolarização, mas que também são necessárias para resolver problemas cotidianos que requerem raciocínio, percepção, domínio de conhecimentos específicos e capacidade de adaptação e assimilação frente a novas informações.

Diversos estudos correlacionam escores obtidos nos testes de inteligência com notas de avaliações das dis- ciplinas escolares ou desempenho em testes acadêmicos, mostrando a relação entre cognição e aprendizagem (Mol \& Wechsler, 2008; Lu, \& cols., 2011; Alloway, \& Gregory, 2013).

Entre as diferentes habilidades, as mais investigadas são a inteligência fluida e a cristalizada. A inteligência fluida é definida como a capacidade de resolver problemas com pouco conhecimento prévio, utilizando habilidades como raciocínio indutivo, sequencial e quantitativo (Schneider, \& McGrew, 2012). Já a inteligência cristalizada se refere ao conhecimento e às habilidades adquiridas a partir da cultura e/ou da educação formal (Schneider, \& McGrew, 2012), como habilidades de conhecimento léxico e compreensão em leitura, relacionados àextensão e profundidade dos conhecimentos adquiridos em experiências de aprendizagem (Carroll, 1993).

Para Flanagan, Ortiz e Alfonso (2013) o desempenho em leitura está associado a habilidades cognitivas distintas, tais como: processamento auditivo, velocidade de processamento, memória de curto prazo, armazenamento e recuperação da memória de longo prazo e inteligência cristalizada. A inteligência fluida e o processamento visual também se relacionam com habilidades de leitura, porém com menor magnitude (Flanagan, Ortiz, Alfonso, \& Mascolo, 2006).

Com relação ao processamento visual, McGrew e Wedling (2010) relatam que embora seja uma das habilidades com correlações mais baixas com leitura, os estudos utilizam apenas baterias mais recentes baseadas no modelo $\mathrm{CHC}$ de inteligência e que a utilização de outros instrumentos poderia indicar magnitudes diferentes de correlação. $O$ modelo $\mathrm{CHC}$ pressupõe uma estrutura fatorial hierárquica da inteligência, a qual é disposta em três níveis de acordo com uma ordem de especialização das habilidades, ou seja, uma dimensão mais geral que representa o fator g (nível III), 16 dimensões amplas tais como inteligência fluida, velocidade de processamento, memória etc. (nível II) e habilidades específicas (nível III) subjacentes à segunda dimensão, como o raciocínio indutivo e sequencial presentes na inteligência fluida (Schneider, \& McGrew, 2012).

Assim como em leitura, a inteligência cristalizada, a velocidade de processamento e a memória de curto prazo estão associadas ao desempenho em matemática. No entanto, habilidades de processamento visual e inteligência fluida estão mais associadas ao desempenho em matemática do que em leitura (Flanagan, \& cols., 2006; McGrew, \& Wedling, 2010). Por sua vez, inteligência fluida e cristalizada, bem como a velocidade de processamento são consistentemente associadas ao desempenho em tarefas que exigem habilidades básicas de aritmética e resolução de problemas (Geray, Hoard, \& Bailey, 2011). Além disso, Mazzoco (2012) aponta para a importância de habilidades visuo-espaciais relacionadas ao processamento de informações visuais no desempenho em aritmética.

De acordo com Mol e Wechsler (2008), o conhecimento acerca do perfil cognitivo do aluno também possibilita ao professor utilizar diferentes estratégias de ensino. Essas autoras investigaram a relação entre habilidades cognitivas como inteligências fluida e cristalizada, memória, raciocínio visuo-espacial e processamento auditivo com notas nas 
disciplinas de Português, Matemática e Ciências em alunos da $1^{\text {a }}$ e $2^{\mathrm{a}}$ séries do Ensino Fundamental. Os resultados indicaram correlações positivas, significativas e magnitude moderada. Primeiramente entre a disciplina de Português e inteligência fluida, cristalizada, memória de trabalho auditiva e de longo prazo, enquanto que a disciplina de Matemática se correlacionou com memória de trabalho auditiva para estímulos numéricos e o desempenho em Ciências se correlacionou também com memória auditiva para sons.

Joly e Istome (2008) investigaram a relação entre inteligência e leitura em 52 crianças a partir dos 9 anos do Ensino Fundamental de uma escola pública no interior de São Paulo. As autoras encontraram correlações positivas, significativas e de magnitude moderada entre o desempenho em leitura e medidas de QI Total, QI Verbal e QI de Execução da Escala Wechsler de Inteligência para Crianças. Diferentes combinações destas variáveis juntamente com os índices de Compreensão Verbal, Organização Perceptual, Resistência a Distração e Velocidade de Processamento predizem 42 a $45 \%$ do desempenho no teste de leitura. Oliveira e Soares (2011) também verificaram que o raciocínio verbal mensurado pela Bateria de Provas de Raciocínio (BPR-5) (Almeida, \& Primi, 2000), prediz quase $11 \%$ das notas em Redação e Português, de alunos entre 10 e 14 anos de idade.

O estudo de Gomes (2010), que avaliou um grupo de estudantes do Ensino Médio de escolas públicas e particulares, mostrou que inteligência fluida explica aproximadamente um terço da variância no desempenho escolar. Segundo Gomes (2010), a capacidade de pensar de forma abstrata e aprender coisas novas envolve estruturas formais do pensamento que influenciam o desempenho escolar. Por outro lado, Primi e cols (2002) avaliaram universitários de diferentes cursos e concluíram que o desempenho acadêmico em Medicina, Engenharia Civil e Matemática esteve mais associado à inteligência fluida. $O$ desempenho acadêmico de alunos dos cursos de Letras e Pedagogia estava mais associado à inteligência cristalizada. Por fim, nos cursos de Administração e Psicologia, tanto inteligência fluida quanto cristalizada estiveram associadas ao desempenho acadêmicodos alunos.

Estes estudos nacionais, realizados nos últimos 12 anos,avaliaram indivíduos a partir de 9 anos de idade.No entanto, ainda são escassos os estudos com crianças mais novas e, além disso, nenhum estudo recente avaliou nível de inteligência e habilidades acadêmicas em crianças dos 6 aos 8 anos. Um instrumento que possibilita a avaliação desta faixa etária é a Bateria de Visualização e Raciocínio da Escala Internacional de Inteligência Leiter-R (Roid, \& Miller, 1997).

A Leiter-R é um teste não-verbal de inteligência, cuja importância está no fato de ser aplicada dos 2 aos 20 anos de idade, abrangendo uma ampla faixa etária, possibilitando comparações em diversas etapas do desenvolvimento. Cabe ressaltar que a Leiter-R é composta por estímulos considerados universais, de modo a minimizar aspectos culturais específicos (Anastasi, \& Urbina, 2000), viabilizando a comparação de diferentes grupos. Além disso, reduz o efeito de variáveis linguísticas, uma vez que não requer inteligência cristalizada. Em função das instruções e formas de respostas não-verbais, também é amplamente utilizada em indivíduos com transtornos do desenvolvimento (Antonio, Mecca, \& Macedo, 2012; Mecca, Orsati, \& Macedo, 2014), condições que se manifestam nas fases iniciais do desenvolvimento e acarretam prejuízos no funcionamento pessoal, social e acadêmico do indivíduo, tais como deficiências intelectuais, transtornos da comunicação, transtorno do espectro autista, transtornos motores, entre outros (Associação Americana de Psiquiatria, 2014).

A Leiter-R foi desenvolvida a partir da Teoria dos Três Estratos sobre a inteligência (Carroll, 1993), avaliando principalmente dois grandes fatores, a Visualização (Gv) e a Inteligência Fluida (Gf). A bateria completa é composta por 10 subtestes, sendo apenas seis necessários ao cálculo do QI. Isto auxilia no tempo demandado para a aplicação, cuja duração é em torno de 30 a 40 minutos (Roid, \& Miller, 1997).O modelo de inteligência dos Três Estratos de Carroll tem sido utilizado em diversos estudos. Um conjunto amplo de evidências sustenta que tanto habilidades específicas quanto habilidades amplas e o fator geralpredizem em torno de 30 a $60 \%$ do desempenho escolar (Gomes, \& Golino, 2012). Além desta característica preditiva, Pereira e Almeida (2010) apontam que testes não-verbais que avaliam a capacidade geral de raciocínio apresentam correlações moderadas, em torno de 0,5 em testes de desempenho acadêmico.

Roid e Miller (1997) apresentam, no manual técnico, estudos de correlação da Leiter-R com testes de desempenho acadêmico como aqueles presentes na Bateria Woodcock-Johnson III (WJ-III).As Correlações de Pearson entre QI Total e os subtestes Broad Reading e Broad Mathematics são de magnitude moderada a alta, indicando uma relação consistente do funcionamento intelectual com as habilidades de aritmética e leitura. Além destes dados descritos por Roid e Miller, são escassos os estudos que investigam a relação ou a predição da inteligência pela Leiter-R em habilidades acadêmicas.

Roberts e cols. (2005) realizaram um estudo cujo objetivo foi avaliar preditores de habilidades acadêmicas em crianças entre 4 e 13 anos de idade com Síndrome do X-Frágil. A Leiter-R foi utilizada para a avaliação do QI Estimado e a WJ para avaliar habilidades acadêmicas. Por se tratar de uma investigação longitudinal, foram utilizadas as médias obtidas a partir das diversas aplicações dos instrumentos. No entanto, os resultados desse estudo não indicaram efeito do QI no aumento dos escores nos subtestes da WJ ao longo das avaliações. Deste modo, outros estudos são necessários para elucidar a relação entre desempenho acadêmico e as habilidades mensuradas pela Leiter-R.

O objetivo do presente estudo foi comparar habilidades cognitivas não-verbais de raciocínio (Gf) e visualização (Gv) avaliadas pela Leiter-R, entre crianças escolas públicas e particulares, dos 6 aos 8 anos de idade. Além disso, buscou-se verificar as relações entre inteligência e habilidades de leitura e aritmética, bem como a capacidade preditiva das habilidades cognitivas no desempenho acadêmico. 


\section{Método}

\section{Participantes}

Foram avaliadas 213 crianças de Ensino Infantil e Fundamental I, entre 6 e 8 anos de idade $(M=6,89 ; D P=0,82)$, sendo 113 pertencentes a escolas públicas $(53,05 \%)$ e 100 a escolas particulares $(46,96 \%)$ da cidade de São Paulo. Os grupos etários foram compostos por 85 crianças com 6 anos (39,90\%), 66 com 7 anos (31\%) e 62 com 8 anos (29,10\%). Participaram do estudo 120 meninos (56,30\%) e 93 meninas $(43,70 \%)$. A amostra foi selecionada por conveniência, de acordo com a disponibilidade e autorização das instituições e responsáveis pelas crianças. Foram excluídas crianças com deficiências sensoriais conhecidas e não corrigidas de acordo com as informações obtidas nas próprias escolas. A Tabela 1 apresenta a caracterização da amostra para as variáveis sexo e idade em função do tipo de escola (pública ou particular).

\section{Instrumentos}

Foram aplicados os seis subtestes da Bateria de Visualização e Raciocínio da Leiter-R que compõem o QI Total para crianças na faixa etária dos 6 aos 8 anos de idade, a saber: Figura-Fundo, Analogias, Formas Completas, Sequências, Padrões Repetidos e Dobra de Papel.

Tabela 1. Descrição da amostra em função do número de participantes nas escolas públicas e particulares.

\begin{tabular}{ccc}
\hline & \multicolumn{2}{c}{ Tipo de escola } \\
\hline Variáveis & Pública & Particular \\
\hline Idades & & \\
\hline 6 & 52 & 33 \\
\hline 7 & 31 & 35 \\
\hline 8 & 30 & 32 \\
\hline Sexo & & \\
\hline Masculino & & \\
\hline Feminino & 58 & 62 \\
\hline
\end{tabular}

O subteste Figura-Fundo avalia habilidades de exploração e discriminação visuais. O objetivo é encontrar o estímulo-alvo em uma prancha na qual estão presentes diversos estímulos distratores. Em Formas Completas avalia-se a capacidade de síntese visual, cuja tarefa é encontrar em uma prancha com diversos estímulos, aquele que representa a figura completa referente ao estímulo-alvo apresentado em fragmentos. No subteste Sequências é avaliada a habilidade de completar sequências que progridem em uma determinada ordem, selecionando os estímulos corretos. Em Padrões Repetidos, os estímulos são apresentados em determinada ordem e o participante deve completar a prancha descobrindo a regra subjacente à sequência, utilizando raciocínio indutivo, enquanto que no subteste Analogias há uma matriz em que faltam estímulos e o indivíduo deve descobrir a relação entre as figuras da matriz para escolher a figura correta que preenche de forma adequada. Por fim, em Dobra de Papel, avalia-se habilidade de manipulação mental de estímulos visuais, uma vez que o objetivo é escolher qual figura representa o estímulo-alvo, caso este estivesse dobrado (Roid, \& Miller, 1997; Mecca, 2010).

Com o objetivo de verificar a relação e predição da Leiter-R no desempenho acadêmico, foram avaliadas habili- dades de aritmética e leitura. Foi aplicada a Prova de Aritmética (Seabra, Montiel, \& Capovilla, 2013), composta por seis subtestes que avaliam competências distintas como escrita por extenso e algébrica de números ditados pelo avaliador, escrita de sequências numéricas crescente e decrescente, relação de grandeza, cálculo envolvendo as quatro operações aritméticas apresentados por escrito e oralmente, bem como resolução de problemas (Dias, \& Seabra, 2013).

A habilidade de leitura foi avaliada pelo Teste de Competência de Leitura de Palavras e Pseudopalavras (TCLPP) que avalia leitura silenciosa de palavras isoladas em crianças do Ensino Fundamental I. Cada item é composto por uma figura e um elemento escrito correspondente. Este pode ser uma palavra ou uma pseudopalavra. O objetivo é julgar se a palavra escrita corresponde à figura ou não (Seabra, \& Capovilla, 2010).

\section{Procedimentos}

O presente estudo foi submetido e aprovado pelo Comitê de Ética da Universidade onde foi realizado (CAAE $n^{\circ}$ 0112.0.272.000-10). A coleta de dados ocorreu nas próprias escolas, após assinatura do Termo de Consentimento Livre 
e Esclarecido pelos responsáveis, durante o período de aula conforme combinado previamente de forma a não prejudicar as atividades escolares dos alunos. Os instrumentos foram aplicados individualmente, em dois encontros com cada criança. No primeiro encontro foi feita a aplicação da Leiter-R (com duração de 30 minutos) e no segundo encontro foi aplicada a Prova de Aritmética (duração de 20 minutos) e o TCLPP (duração de 10 a 15 minutos).

O desempenho na Leiter-R foi comparado entre crianças de escola pública e particular, separadamente por faixa etária. Foram realizadas análises de Correlação de Pearson entre os instrumentos utilizados, bem como análises de Regressão Linear para verificar o valor preditivo da Leiter-R no desempenho em aritmética e leitura. Para análise dos resultados foi utilizado o programa Statistical Package for Social Sciences ${ }^{\circledR}$ versão 18.0 para Windows (SPSS Inc).

\section{Resultados}

Primeiramente comparou-se o desempenho entre crianças de escolas públicas e particulares na Leiter-R. Os dados referentes à média, desvio-padrão, ANOVA, significância e tamanho de efeito são apresentados na Tabela 2.

Tabela 2. Comparação entre escolas públicas e particulares na amostra total e por faixa etária.

\begin{tabular}{|c|c|c|c|c|c|}
\hline Subtestes & Idade & Escola Pública & Escola Particular & $\mathbf{F}$ & d \\
\hline \multirow[t]{4}{*}{ Figura-Fundo } & 6 & $15,35( \pm 3,71)$ & $17,3( \pm 2,60)$ & $6,969^{*}$ & 0,59 \\
\hline & 7 & $18,71( \pm 3,16)$ & $18,74( \pm 3,48)$ & 0,002 & 0,01 \\
\hline & 8 & $17,63( \pm 2,89)$ & $19,88( \pm 3,62)$ & $4,332^{*}$ & 0,68 \\
\hline & Total & $16,88( \pm 3,64)$ & $18,47( \pm 3,35)$ & $10,933^{*}$ & 0,45 \\
\hline \multirow[t]{4}{*}{ Analogias } & 6 & $4,27( \pm 2,33)$ & $5,45( \pm 2,46)$ & $4,983^{*}$ & 0,70 \\
\hline & 7 & $5,16( \pm 2,51)$ & $7,89( \pm 5,89)$ & $5,693^{*}$ & 0,61 \\
\hline & 8 & $5,93( \pm 2,95)$ & $7,44( \pm 3,38)$ & 3,447 & 0,44 \\
\hline & Total & $4,96( \pm 2,63)$ & $6,94( \pm 4,31)$ & $16,819^{*}$ & 0,56 \\
\hline \multirow[t]{4}{*}{ Formas Completas } & 6 & $20,88( \pm 4,47)$ & $24,24( \pm 5,16)$ & $10,031^{*}$ & 0,71 \\
\hline & 7 & $26,32( \pm 4,89)$ & $28,11( \pm 3,30)$ & 3,098 & 0,43 \\
\hline & 8 & $27,00( \pm 4,43)$ & $27,81( \pm 3,86)$ & 0,594 & 0,21 \\
\hline & Total & $24,00( \pm 5,39)$ & $26,74( \pm 4,48)$ & $15,985^{*}$ & 0,55 \\
\hline \multirow[t]{4}{*}{ Sequências } & 6 & $9,96( \pm 4,94)$ & $15,39( \pm 7,14)$ & $17,171^{*}$ & 0,92 \\
\hline & 7 & $16,65( \pm 7,93)$ & $22,66( \pm 8,38)$ & $8,891^{*}$ & 0,98 \\
\hline & 8 & $16,93( \pm 7,24)$ & $22,63( \pm 8,94)$ & $7,521^{*}$ & 0,70 \\
\hline & Total & $13,65( \pm 7,30)$ & $20,25( \pm 8,79)$ & $35,821^{*}$ & 0,82 \\
\hline \multirow[t]{4}{*}{ Padrões Repetidos } & 6 & $11,62( \pm 4,65)$ & $15,42( \pm 4,99)$ & $12,778^{*}$ & 0,79 \\
\hline & 7 & $16,42( \pm 4,50)$ & $17,86( \pm 4,46)$ & 1,694 & 0,32 \\
\hline & 8 & $15,87( \pm 3,46)$ & $17,31( \pm 3,93)$ & 2,345 & 0,39 \\
\hline & Total & $14,07( \pm 4,85)$ & $16,88( \pm 4,56)$ & $18,893^{*}$ & 0,60 \\
\hline \multirow[t]{4}{*}{ Dobra de Papel } & 6 & $3,87( \pm 1,70)$ & $5,72( \pm 1,70)$ & $23,392^{*}$ & 1,09 \\
\hline & 7 & $5,32( \pm 1,57)$ & $6,12( \pm 1,81)$ & 3,506 & 0,47 \\
\hline & 8 & $5,97( \pm 1,77)$ & $6,69( \pm 1,99)$ & 2,257 & 0,38 \\
\hline & Total & $4,83( \pm 1,91)$ & $6,17( \pm 1,86)$ & $26,738^{*}$ & 0,71 \\
\hline \multirow[t]{4}{*}{ Leiter-R Total } & 6 & $65,94( \pm 14,51)$ & $83,36 \pm(16,96)$ & $25,501^{*}$ & 1,12 \\
\hline & 7 & $88,58( \pm 17,42)$ & $101,03( \pm 19,06)$ & $7,596^{*}$ & 0,68 \\
\hline & 8 & $89,33( \pm 14,82)$ & $101,25( \pm 19,84)$ & $7,100^{*}$ & 0,68 \\
\hline & Total & $78,37( \pm 19,15)$ & $95,27( \pm 20,28)$ & $39,099^{*}$ & 0,86 \\
\hline
\end{tabular}

Tamanho de efeito pequeno $(0.20 \leq d<0.50)$; médio $(0.50 \leq d<0.80)$ e grande $(d \geq 0.80)$.

${ }^{*} p \leq 0,05$. 
Os dados apresentados na Tabela 2 mostraram que de modo geral há diferenças em todos os subtestes e nos escores totais entre crianças de escolas públicas e particulares. No entanto, os resultados indicam que estas diferenças não são observadas em todos os subtestes de acordo com cada faixa etária. Aos 6 anos de idade, observaram-se diferenças estatisticamente significativas entre escolas públicas e particulares para todos os subtestes e a pontuação total. Por outro lado, observam-se diferenças estatisticamente significativas entre crianças de escolas públicas e particulares com 7 anos de idade nos subtestes Sequências e Analogias, bem como na pontuação total. Já paraos subtestes FiguraFundo, Formas Completas, Padrões Repetidos e Dobra de Papel não foram encontradas diferenças. Por fim, aos 8 anos, foram verificadas diferenças significativas entre as escolas nos subtestes Figura-Fundo e Sequências, assim como no total, enquanto que nesta faixa etária não foram observadas diferenças estatisticamente significativas entre as escolas para os subtestes Analogias, Formas Completas, Padrões Repetidos e Dobra de Papel.
Em função das diferenças observadas entre crianças pertencentes a escolas públicas e particulares, com maiores desempenhos destas, foram realizadas comparações entre as faixas etárias considerando separadamente o tipo de escola. Os dados obtidos a partir ANOVA para as escolas públicas são apresentados na Tabela 3.

Conforme os dados apresentados na Tabela 3, observam-se diferenças estatisticamente significativas entre as faixas etárias nas crianças de escolas públicas ( $p \leq 0,01)$. Análises Post Hoc com correção de Bonferroni mostraram diferenças significativas entre as crianças de 6 e 7 anos para a maioria dos subtestes e nos escores totais, com exceção do subteste Analogias onde não foram observadas diferenças entre estes grupos. Já entre as idades de 6 e 8 anos, foram observadas diferenças significativas nos desempenhos de todos os subtestes bem como nos escores totais. Não foram verificadas diferenças significativas entre as crianças de 7 e 8 anos pertencentes a escolas públicas em todos os subtestes, assim como nos escores totais. Na Tabela 4 são apresentados os dados referentes às crianças de escolas particulares.

Tabela 3. Comparação entre as faixas etárias nos estudantes de escolas públicas.

\begin{tabular}{ccccc}
\hline Subteste & $\mathbf{6}$ & $\mathbf{7}$ & $\mathbf{8}$ & $\mathbf{F}$ \\
Figura-fundo & $15,35( \pm 3,71)$ & $18,71( \pm 3,16)$ & $17,63( \pm 2,89)$ & $10,723^{*}$ \\
Analogias & $4,27( \pm 2,33)$ & $5,16( \pm 2,51)$ & $5,93( \pm 2,95)$ & $4,149^{*}$ \\
Formas Completas & $20,88( \pm 4,47)$ & $26,32( \pm 4,89)$ & $27,00( \pm 4,43)$ & $22,329^{*}$ \\
Sequências & $9,96( \pm 4,94)$ & $16,65( \pm 7,93)$ & $16,93( \pm 7,24)$ & $15,453^{*}$ \\
Padrões Repetidos & $11,62( \pm 4,65)$ & $16,42( \pm 4,50)$ & $15,87( \pm 3,46)$ & $15,524^{*}$ \\
Dobra de Papel & $3,87( \pm 1,70)$ & $5,32( \pm 1,57)$ & $5,97( \pm 1,77)$ & $16,592^{*}$ \\
Total & $65,94( \pm 14,51)$ & $88,58( \pm 17,42)$ & $89,33( \pm 14,82)$ & $31,193^{*}$ \\
\hline
\end{tabular}

${ }^{*} p \leq 0,01$.

Tabela 4. Comparação entre as faixas etárias nos estudantes de escolas particulares.

\begin{tabular}{ccccc}
\hline Subteste & $\mathbf{6}$ & $\mathbf{7}$ & $\mathbf{8}$ & $\mathbf{F}$ \\
Figura-Fundo & $17,3( \pm 2,60)$ & $18,74( \pm 3,48)$ & $19,88( \pm 3,62)$ & $3,449^{*}$ \\
Analogias & $5,45( \pm 2,46)$ & $7,89( \pm 5,89)$ & $7,44( \pm 3,38)$ & $3,138^{*}$ \\
Formas Completas & $24,24( \pm 5,16)$ & $28,11( \pm 3,30)$ & $27,81( \pm 3,86)$ & $8,879^{*}$ \\
Sequências & $15,39( \pm 7,14)$ & $22,66( \pm 8,38)$ & $22,63( \pm 8,94)$ & $8,667^{*}$ \\
Padrões Repetidos & $15,42( \pm 4,99)$ & $17,86( \pm 4,46)$ & $17,31( \pm 3,93$ & 2,712 \\
Dobra de Papel & $5,72( \pm 1,70)$ & $6,12( \pm 1,81)$ & $6,69( \pm 1,99)$ & 2,235 \\
Total & $83,36 \pm(16,96)$ & $101,03( \pm 19,06)$ & $101,25( \pm 19,84)$ & $10,029^{*}$ \\
\hline$*_{p}<0,05$. & & &
\end{tabular}


Análises de variância entre as faixas etárias de alunos de escolas particulares revelaram que há diferenças estatisticamente significativas entre os grupos na maioria dos subtestes e nos escores totais, com aumento da pontuação principalmente dos 6 para 7 anos de idade. No entanto, tais diferenças não foram encontradas nos subtestes Padrões Repetidos e Dobra de Papel.

Comparação de pares por correção de Bonferroni mostrou diferenças estatisticamente significativas entre crianças de 6 e 7 anos apenas nos subtestes Formas Completas e Sequências, bem como nos escores totais. Observou-se uma tendência de diferença para o subteste Analogias $(p=0,059)$ entre estas faixas etárias. Já entre as crianças entre 6 e 8 anos de idade, as diferenças significativas foram observadas nos subtestes Figura-Fundo, Formas Completas, Sequências e escores totais. Por fim, entre as crianças de 7 e 8 anos, os resultados permaneceram idênticos aos apresentados na amostra total e nas crianças de escolas públicas, ou seja, não foram observadas diferenças significativas entre estas faixas etárias em nenhum dos subtestes, assim como nos escores totais nas crianças de escolas particulares.

Com objetivo de verificar a relação entre habilidades cognitivas não-verbais avaliadas pela Leiter- $R$ e habilidades acadêmicas, foram realizadas análise de Correlação de Pe- arson com a Prova de Aritmética e o TCLPP. Os resultados obtidos mostraram correlações positivas, significativas e de magnitude moderada entre o desempenho total obtido na Leiter-R e o teste de leitura TCLPP $(r=0,56 ; p<0,01)$, bem como entre a Leiter- $R$ e a Prova de Aritmética ( $r=0,51$; $p<0,01)$. A Tabela 5 apresenta as correlações obtidas entre os subtestes da Leiter-R, a Prova de Aritmética e o TCLPP.

De acordo com os dados apresentados na Tabela 5 , foram observadas correlações positivas, significativas e de magnitude moderada entre a Prova de Aritmética e os subtestes Sequencias, Analogias e Dobra de Papel, além de uma tendência para o subteste Formas Completas. O TCLPP também se correlacionou com os subtestes Sequências, Analogias e Dobra de Papel, assim como com Padrões Repetidos.

Em seguida foi realizada análise de regressão linear para verificar a predição da Leiter-R nas provas que avaliam desempenho acadêmico. Os achados encontram-se sumariados na Tabela 6 .

A análise de regressão linear apontou para um coeficiente de determinação no valor de 0,242 para aritmética. Isto significa que o desempenho no teste de inteligência Leiter-R prediz $24,2 \%$ do desempenho em aritmética. Já o coeficiente de determinação para o TCLPP foi de 0,306 , indicando que $30,6 \%$ da variância no desempenho do TCLPP podem ser explicados pela inteligência.

Tabela 5. Correlações obtidas entre os subtestes da Leiter-R, a Prova de Aritmética e o TCLPP.

\begin{tabular}{ccc}
\hline Subtestes & Prova de Aritmética & TCLPP \\
\hline Figura-Fundo & 0,20 & 0,22 \\
\hline Formas Completas & 0,26 & 0,24 \\
\hline Sequências & $0,52^{* *}$ & $0,55^{* *}$ \\
\hline Padrões Repetidos & 0,17 & $0,49^{* *}$ \\
\hline Analogias & $0,46^{* *}$ & $0,40^{* *}$ \\
\hline Dobra de Papel & $0,34^{*}$ & $0,26^{*}$ \\
\hline
\end{tabular}

${ }^{*}$ Correlação é significativa ao nível de 0,05 .

** Correlação é significativa ao nível de 0,01.

Tabela 6. Sumário do modelo oriundo da Regressão Linear.

\begin{tabular}{ccccccc}
\hline Modelo & $\mathrm{R}$ & $\mathrm{R}^{2}$ & $\begin{array}{c}\mathrm{R}^{2} \\
\text { Ajustado }\end{array}$ & $\begin{array}{c}\text { Erro Padrão de } \\
\text { Estimativa }\end{array}$ & $\mathrm{F}$ & $\mathrm{p}$ \\
\hline Aritmética & 0,507 & 0,257 & 0,242 & 9,106 & 16,930 & 0,000 \\
\hline TCLPP & 0,565 & 0,319 & 0,306 & 6,280 & 25,749 & 0,000 \\
\hline
\end{tabular}




\section{Discussão}

De acordo com os resultados obtidos a partir da comparação do desempenho entre crianças de escolas públicas e particulares, observou-se que crianças pertencentes a instituições privadas apresentam maiores médias em relação a crianças de instituições públicas. Este padrão foi observado em todos os subtestes, com diferenças estatisticamente significativas. Esses achados vão ao encontro com o que se observa em outros instrumentos não-verbais que avaliam inteligência na infância, como as Matrizes Progressivas Coloridas de Raven (Angelini, \& cols., 1999) e a Escala de Maturidade Mental Colúmbia (Alves,\& Duarte, 2001), refletindo a composição de normas diferenciadas para as diferentes escolas. Tais diferenças também são verificadas em instrumentos destinados aos adolescentes de Ensino Fundamental II e Ensino Médio, tal como a Bateria de Provas de Raciocínio (BPR-5), que avalia raciocínio verbal, abstrato, numérico, espacial e mecânico (Almeida, \& Primi, 2000).

Teoricamente, os testes não-verbaiscomo a Leiter-R e o Raven avaliam aspectos da inteligência que não estão associados a um conhecimento formal prévio ou a habilidades adquiridas culturalmente (Anastasi, \& Urbina, 2000). Seguindo esta premissa, o desempenho não poderia estar relacionado a qualquer influência ambiental. No entanto, estudos apontam que testes como o Raven, que avaliam a inteligência fluida, apresentam ganhos à medida que a cultura evolui (Flynn, 2009). Esta influência ambiental pode ser observada nos resultados do presente estudo à medida que o ambiente influenciou o desempenho das crianças que pertencem a diferentes tipos de escolas.

Além disso, também foram identificadas diferenças no padrão de desempenho entre escolas públicas e particulares quando as comparações foram realizadas de acordo com cada faixa etária. Os resultados mostraram que diferenças estatisticamente significativas entre escolas públicas e particulares em todos os subtestes e pontuação total na faixa etária dos 6 anos, sendoque menores médias foram apresentadas por alunos de escolas públicas. Aos 7 anos de idade, as diferenças ocorreram somente nos subtestes Analogias e Sequências, também com médias inferiores no grupo de escola pública, enquanto que aos 8 anos, as diferenças entre as escolas estão nos subtestes Figura-Fundo e Sequências.

Portanto, as diferenças observadas entre os grupos de escolas públicas e particulares não se assemelham entre as diversas idades, uma vez que maiores discrepâncias ocorrem entre as crianças com 6 anos de idade. Estes resultados são diferentes daqueles observados por Alves e Duarte (2001) com a Escala de Maturidade Mental Columbia, em que o grupo de escolas públicas apresentou desempenho inferior em todas as faixas etárias quando comparado as escolas particulares.

No entanto, as diferenças observadas nesta faixa etária podem estar relacionadas a uma discrepância nos anos de escolarização ou série escolar. As crianças com 6 anos de escolas públicas pertenciam ao último ano do Ensi- no Infantil, enquanto que as crianças da mesma faixa etária de escolas particulares, pertenciam ao $1^{\circ}$ ano do Ensino Fundamental. Almeida, Lemos, Guisande e Primi (2008) discutem a relevância das variáveis idade e escolaridade no desempenho em testes de inteligência. De acordo com esses autores, o desempenho em testes na infância se justificaria mais pela faixa etária do que pela série escolar, uma vez que há uma associação maior entre estas variáveis em função da maior influência da maturação do sistema nervoso neste período. Nesse sentido, optou-se por comparar estes grupos mesmo com cerca de um ano de diferença no tempo de escolarização.

Ao comparar os desempenhos das diferentes faixas etárias em cada tipo de escola separadamente, foram observados padrões diferentes entre as crianças de escolas públicas e particulares. As crianças com 6 anos de idade nas escolas públicas apresentam menores médias em relação as crianças com 7 anos em todos os subtestes, com exceção de Analogias, ao passo que nas escolas particulares, menores pontuações aos 6 anos são estatisticamente significativas somente nos subtestes Formas Completas e Sequências, enquanto que nos outros subtestes o desempenho é semelhante. Neste sentido, existem mais diferenças nos alunos de escolas públicas do que em alunos de escolas particulares entre as faixas etárias.

Ao comparar crianças entre 6 e 8 anos de idade, observam-se diferenças estatisticamente significativas, sendo que nas escolas públicas as diferenças são observadas em todos os subtestes. No entanto, nas escolas particulares, o desempenho aos 6 anos de idade é inferior aos 8 anos apenas nos subtestes Figura-Fundo, Formas Completas e Sequências. Observou-se que, nas escolas particulares, os subtestes Formas Completas e Sequências foram mais sensíveis para diferenciar as idades.Por fim, tanto em escolas públicas quanto em escolas particulares, as crianças com 7 e 8 anos não apresentaram diferenças no desempenho tanto nos subtestes específicos quanto na pontuação total. Sendo assim, verifica-se que de modo geral, independente do tipo de escola, as habilidades relacionadas ao fator $\mathrm{Gv}$, como discriminação, exploração e integração visual não aumentam de forma significativa dos 7 para os 8 anos. 0 mesmo pode ser observado nas habilidades relacionadas à Gf, como raciocínio sequencial e indução de relações.

A heterogeneidade observada nos resultados a partir das diferentes faixas etárias e do tipo de escola pode decorrer em função da fase de desenvolvimento em que as crianças se encontram. A capacidade de raciocínio demandada pelo subteste Analogias, por exemplo, se desenvolve a partir dos 6 anos de idade (Papalia, Olds, \& Feldman, 2009). As diferenças podem não ser significativas entre crianças de 6 a 8 anos em ambos os tipos de escola, por se tratar de uma habilidade ainda incipiente, ou em desenvolvimento. Neste sentido, os resultados do presente estudo mostraram que o tipo de ambiente pode ter mais influência no desempenho em tarefas de raciocínio indutivo para estímulos visuo-espaciais do que a idade cronológica, em se tratando de crianças pertencentes aos anos iniciais do Ensino Fundamental. 
No que tange à relação entre leitura, aritmética e o desempenho na Leiter-R, as correlações positivas, significativas e de magnitude moderada corroboram resultados prévios descritos na literatura com indivíduos de diferentes faixas etárias (Primi, \& Almeida, 2000; Mol, \& Wechsler, 2008). Estes achados corroboram tanto a relação entre aspectos não-verbais da inteligência e aritmética (Taub, Floyd, Keith, \& McGrew, 2008; Flanagan, \& cols, 2013) e leitura (Mol, \& Wechsler, 2008; Joly, \& Istome, 2008), embora, de modo geral, o desempenho em leitura seja mais relacionado com medidas verbais, indicativas de inteligência cristalizada e processamento auditivo (Flanagan, \& cols., 2006; Joly, \& Istome, 2008; Alloway, \& Gregory, 2013). Isto justifica a magnitude moderada encontrada no presente estudo, assim como em estudos anteriores. Apenas uma parte da variância do desempenho acadêmico está relacionada com a inteligência fluida (Flanagan, \& cols., 2013) enquanto que há outra parte relacionadaa outros fatores, tais como a inteligência cristalizada (Primi, \& cols., 2002; Mol, \& Wechsler, 2008; Joly, \& Istome, 2008; Oliveira, \& Soares, 2011).

Ao analisar os subtestes específicos da Leiter-R, foram verificadas correlações de magnitude moderadaentre Sequências, Analogias, Dobra de Papel e a Prova de Aritmética, o que parece indicar que habilidades matemáticas de compreensão das propriedades dos números e das operações que podem ser realizadas com eles estão relacionadas a habilidades cognitivas visuo-espaciais, raciocínio sequencial e analógico, bem como manipulação mental de estímulos visuais. Uma das habilidades aritméticas avaliadas é o sequenciamento numérico crescente e decrescente. McGrew (2009) relata que o raciocínio sequencial é uma habilidade subjacente à inteligência fluida. Neste sentido,reflete uma maior complexidade cognitiva de processos envolvidos em execução de tarefas. Entre eles estão os processos dedutivos relacionados a capacidade de raciocinar e obter conclusões a partir de premissas gerais mais específicas, como deduzir a resolução dos problemas através dos enunciados de matemática.

Os mesmos subtestes foram correlacionados com o TCLPP, além do subteste Padrões. Desta forma, as mesmas habilidades citadas anteriormente, juntamente com a capacidade de descobrir regras subjacentes a uma sequência de estímulos se relacionam com a habilidade de leitura de palavras. Estes achados corroboram estudo anterior que indica habilidades de sequenciamento relacionadas ao desempenho em leitura e escrita (Capovilla, Gutschow, \& Capovilla, 2004).

Além das relações observadas entre os subtestes da Leiter-R com as provas de desempenho acadêmico, foi verificada a predição daquela em relação a estas provas. Os resultados oriundos da análise de regressão linear mostraram que habilidades de processamento visual (Gv) e raciocínio fluido (Gf) predizem $24 \%$ do desempenho em aritmética e $30 \%$ do desempenho em leitura. A variância não explicada por essas habilidades cognitivas podem estar relacionadas a outras variáveis como idade, série escolar, motivação e funções executivas, conforme apontam outros estudos (Clark, Pritchard, Verena, Woodward, \& Lianne, 2010; Lu, \& cols., 2011; Primi, Couto, Almeida, Guisande, \& Miguel, 2012).
Estes dados replicam achados previamente descritos na literatura que apontam para a inteligência geral como uma importante variável preditora de desempenho acadêmico (Cruz, 2008; Rowe, Miller, Ebenstein, \& Thompson, 2012).

O presente estudo teve por objetivo compreender a relação entre variáveis presentes no contexto educacional, tais como o desempenho acadêmico em leitura e aritmética, assim como o tipo de escola a qual a criança pertence, com habilidades cognitivas não-verbais. Assim, ressalta-se a importância de considerar determinados aspectos, como o desempenho acadêmico e o tipo de escola, nas práticas de avaliação de inteligência. Primeiramente, o tipo de escola a qual a criança pertence, uma vez que escolas públicas e particulares refletem condições socioeconômicas distintas (IBGE, 2012). Além disso, habilidades cognitivas nãoverbais tais como manipulação mental de estímulos visuais, raciocínio analógico, indutivo e sequencial se relacionam e predizem o desempenho acadêmico tanto em demandas de leitura como aritmética. Neste sentido, a realização de atividades que estimulem estas habilidades pode influenciar no desempenho em aritmética e leitura.

\section{Referências}

Alloway, T. P., \& Gregory, D. (2013). The predictive ability of IQ and Working Memory scores in literacy in an adult population. International Journal of Educational Research, 57, 51-56.

Almeida, L. S., \& Primi, R. (2000). Bateria de Provas de Raciocínio BPR-5. São Paulo: Casa do Psicólogo.

Almeida, L. S., Lemos, G., Guisande, M. A., \& Primi, R. (2008). Inteligência, Escolarização e Idade: Normas por Idade ou Série Escolar? Avaliação Psicológica, 7(2), 117-125.

Alves, I., \& Duarte, J. (2001). Escala de Maturidade Mental Columbia - Padronização Brasileira. São Paulo: Casa do Psicólogo.

American Psychiatric Association [APA]. (2013). Diagnostic and statistical manual of mental disorders - DSM-V. Washington: APA.

Anastasi, A., \& Urbina, S. (2000). Testagem Psicológica. Porto Alegre: Artmed.

Angelini, A. L., Alves, I. C. B., Custódio, E. M., Duarte, W. F., \& Duarte, J. L. (1999). Matrizes Progressivas Coloridas de Raven: Escala Especial. São Paulo: CETEPP.

Antonio, D. A. M., Mecca, T. P., \& Macedo, E. C. (2012). O uso do teste não-verbal Leiter-R na avaliação de inteligência em distúrbios do desenvolvimento. Cadernos de Pós-graduação em Distúrbios do Desenvolvimento, 12(2), 9-15.

Capovilla, A. G. S., Gutschow, C. R. D., \& Capovilla, F. C. (2004). Habilidades cognitivas que predizem competência de leitura e escrita. Psicologia: Teoria e Prática, 6(2), 13-26. 
Carneiro, P., Meghir, C., \& Parey, M. (2007). Maternal education, home environments and the development of children and adolescents. IZA Discussion Paper, 3072. Recuperado: jan. 2014. Disponível: http://ftp.iza.org/dp3072.pdf.

Carneiro, P., Meghir, C., \& Parey, M. (2013). Maternal education, home environments and the development of children and adolescents. Journal of the European Economic Association, 11(1), 123-160.

Carroll, J. B. (1993). Human Cognitive Abilities: A Survey of Factor Analytic Studies. New York: Cambridge University Press.

Chevalier, A., Harmon, C., O'Sullivan, V., \&Walker, I. (2005). The impact of parental income and education on the schooling of their children. The Institute for Fiscal Studies. Disponível: http://www.ifs. org.uk/wps/wp0505.pdf.

Clark, C. A. C., Pritchard, V. E., \& Woodward, L, J. (2010).Preschool executive functioning abilities predict early mathematics achievement. Developmental Psychology, 46(5), 1176-1191.

Cruz, M. B. Z. (2008). Estudo de validade e precisão da bateria de provas de raciocínio infantil-BPR-5I. Dissertação de Mestrado, Universidade São Francisco, Itatiba-SP.

Dias, N. M., \& Seabra, A. G. (2013). Evidências de validade e fidedignidade da Prova de Aritmética. Em N. M. Dias, \& A. G. Seabra (Orgs.), Avaliação Neuropsicológica Cognitiva Volume 3: Leitura, escrita e aritmética. (pp. 85-91). São Paulo: Memnon Edições Científicas,

Flanagan, D. P., Ortiz, S. O., Alfonso, V. C., \& Mascolo, J. T. (2006). The Achievement Test Desk Reference (ADRT): A Guide to Learning Disability Identification. Boston: Allyn \& Bacon.

Flanagan, D. P., Ortiz, S. O., \& Alfonso, V. C. (2013). Essentials of Cross-Battery Assessment ( $3^{\text {rd }}$ edition).New Jersey: John Wiley\& Sons, Inc.

Flynn, J. R. (2009). O que é Inteligência? Além do Efeito Flynn. Porto Alegre: Artmed.

Geary, D. C., Hoard, M. K., \& Bailey, D. H. (2011). How SLD manifests in mathematics. Em D. P. Flanagan, \& V. C. Alfonso (Orgs.), Essentials of Specific Learning Disability Identification (pp. 43-64). Hoboken, NJ: Wiley.

Gomes, C. M. A.(2010). Avaliando a Avaliação Escolar: Notas Escolares e Inteligência Fluida. Psicologia em Estudo, Maringá, 15(4), 841-849.

Instituto Brasileiro de Geografia e Estatística [IBGE]. (2012). Diretoria de Pesquisas, Coordenação de População e Indicadores Sociais. Pesquisa Nacional de Saúde do Escolar.

Joly, M. C. R. A., \& Istome, A. C. (2008). Compreensão em leitura e capacidade cognitiva: estudo de validade do teste Cloze básico-
MAR. Psic:Revista da Vetor Editora, 9(2), 219-228.

Lu L, Weber H. S, Spinath F. M., \& Shi J. (2011). Predicting school achievement from cognitive andnon-cognitive variables in a Chinese sampleof elementary school children. Intelligence,39(2-3), 130-140.

McGrew, K. S. (2009). CHC theory and the human cognitive abilities project: Standing on the shoulders of the giants of psychometric intelligence research. Intelligence, 37(1), 1-10.

McGrew, K. S., \& Wedling, B. J. (2010).Cattell-Horn-Carroll cognitiveachievement relations: What we have learned from the past 20 years of research. Psychology in the Schools, 47, 651-675.

Mecca, T. P. (2010). Tradução, adaptação, fidedignidade e evidências de validade da Bateria de Visualização e Raciocínio da Leiter International Performance Scale Revised. Dissertação de Mestrado, Universidade Presbiteriana Mackenzie, São Paulo-SP.

Mecca, T. P., Orsati, F. T., \& Macedo, E. C. (2014). Inteligência e Transtornos do Desenvolvimento. Em A. G. Seabra., J. A. Laros., E. C. Macedo, \& A. Neander (Orgs.),Inteligência e Funções Executivas: Avanços e Desafios para Avaliação Neuropsicológica (pp. 112-134). São Paulo: Memnon Edições Científicas.

Mól, D. A. R., \& Wechsler, S. M. (2008). Avaliação de crianças com indicação de dificuldades de aprendizagem pela bateria Woodcock-Johnson III. Psicologia Escolar e Educacional, 12(2), 391-399.

Oliveira, M. B., \& Soares, A. B. (2011). Auto-eficácia, raciocínio verbal e desempenho escolar em estudantes. Psicologia: Teoria e Pesquisa, 27(1), 33-39.

Papalia, D. E., Olds, S. W., \& Feldman, R. D. (2009). Desenvolvimento Humano. São Paulo: McGraw-Hill.

Pereira, M., \& Almeida, L. (2010). Predição do rendimento académico no final do ensino secundário na base dos testes de QI na infância. Revista Galego-Portuguesa de Psicoloxía e Educación, 18(1), 239-249.

Primi, R., Santos, A. D., \& Vendramini, C. M. (2002). Habilidades básicas e desempenho acadêmico em universitários ingressantes. Estudos de Psicologia, 7(1), 47-55.

Primi, R., Couto, G., Almeida, L. S., Guisande, M. A., \& Miguel, F. K. (2012).Intelligence, Age and Schooling: Data from the Battery of Reasoning Tests (BRT-5). Psicologia: Reflexão e Crítica, 25(1), 79-88.

Roberts, J. E., Schaaf, J. M., Skinner, M., Wheeler, A., Hooper, S., Hatton, D. D. \& Bailey Jr, D. B. (2005). Academic Skills of Boys with Fragile X Syndrome: Profiles and Predictors. American Journal on Mental Retardation, 110(2), 107-120. 
Roid, G. H., \& Miller, L. J. (1997). Leiter International Performance Scale-Revised. Wood Dale, IL: Stoelting.

Rowe, E. W., Miller, C., Ebenstein, L. A., \& Thompson, D. F. (2012). Cognitive predictors of reading and math achievement among gifted referrals. School Psychology Quarterly, 27(3), 144-153.

Schneider, W. J., \& McGrew, K. S. (2012). The Cattell-Horn-Carroll modelofintelligence. Em D. P. Flanagan, \& P. L. Harrison (Orgs.), Contemporary intellectual assessment: Theories, Tests, and Issues (3rd ed., pp. 99-144). New York, NY: The Guilford Press.

Seabra, A. G., \& Capovilla, F. C. (2010). Teste de Competência de Leitura de Palavras e Pseudopalavras. São Paulo: Memnon Edições Científicas.

Seabra, A. G., Montiel, J. M., \& Capovilla, F. C. (2013). Prova de Aritmética. EmN. M. Dias,\& A. G. Seabra (Orgs.), Avaliação
Neuropsicológica Cognitiva: Leitura, escrita e aritmética (pp.97104). São Paulo: Memnon Edições Científicas.

Stock, P., Desoete, A., \& Roeyers, H. (2009). Predicting Arithmetic Abilities: The Role of Preparatory Arithmetic Markers and Intelligence. Journal of Psychoeducational Assessment, 27, 237251.

Taub, G. E., Floyd, R. G., Keith, T. Z., \& McGrew, T. Z. (2008). Effects of general and broad cognitive abilities on mathematics achievement. School Psychology Quarterly, 23, 187-198.

Vista, A.D., \& Grantham, T. C. (2010). Effects of Parental Education Level on Fluid Intelligence of Philippine Public School. Journal of Psychoeducational Assessment, 28(3), 236-248.

\section{Sobre os autores}

Tatiana Pontrelli Mecca (tati.mecca@gmail.com)

Psicóloga, Doutora em Distúrbios do Desenvolvimento. Docente do Centro UniversitárioFieo - UNIFIEO

Tatiana Abraão Jana (tataarabella@uol.com.br)

Estudante de Psicologia na Universidade Presbiteriana Mackenzie

Mário Rodrigues Simões (simoesmr@fpce.uc.pt)

Psicólogo, Doutor em Avaliação Psicológica. Docente da Universidade de Coimbra

Elizeu Coutinho de Macedo (elizeumacedo@uol.com.br)

Psicólogo, Doutor em Psicologia Experimental. Docente da Universidade Presbiteriana Mackenzie.

Apoio financeiro: Agradecimentos à FAPESP, à CAPES e ao CNPq. 
